\title{
Effects of Neck and Trunk Stabilization Exercise on Balance in Older Adults
}

\author{
Gui-bin Song ${ }^{1}$, Eun-Cho Park ${ }^{2}$ \\ 'Department of Physical Therapy, Yeungnam University College, Daegu; ${ }^{2}$ Department of Physical therapy, College of Rehabilitation Science Daegu \\ University, Gyeongsan, Korea
}

Purpose: This study was conducted to evaluate the effects of neck and trunk stabilization exercise on static and dynamic balance in older adults.

Methods: A total of 30 older adults participated in this study. Participants were randomly assigned to the neck and trunk stabilization exercise group (NTSG) $(n=15)$ or the trunk stabilization exercise group (TSG) $(n=15)$. The NTSG performed a trunk stabilization exercise added to a neck stabilization exercise that included biofeedback. Both groups received training for 30 minutes per day three times per week for eight weeks. The anterior, posterior limit of stability and sway length was used to measure static balance ability, while the timed up and go (TUG) test was used to measure dynamic balance ability.

Results: Participants showed significant differences in sway length, anterior limit of stability, posterior limit of stability, and the results of the TUG test between their pre- and post mediation evaluations $(p<0.05)$. The NTSG showed a more significant increase than the TSG $(p<0.05)$.

Conclusion: According to the results of this study, both exercises effectively improved static and dynamic balance ability. However, the neck and trunk stabilization exercise is more efficient for increasing the balance ability of older adults.

Keywords: Neck and trunk stabilization exercise, Balance, Older adults

\section{INTRODUCTION}

Recent advancements in medicine and economic development have increased the average life expectancy, which has led to an increase in the proportion of elderly people in the population. As the number of elderly people has increased, the aging of the population has become an important social issue. Korea has already become an aging society; in 2000, elderly people accounted for $7.2 \%$ of the total population, and this figure is expected to reach $14.4 \%$ by $2019 .{ }^{1} \mathrm{An}$ aging population entails both social and psychological problems; many elderly people feel socially isolated and face economic hardships while suffering physical conditions, such as those resulting from chronic or degenerative diseases, and mental conditions, such as depression and a sense of isolation. ${ }^{2}$ Function degradation of the elderly causes injuries in them, which take a great economic toll not

Received Jul 11, 2016 Revised Aug 11, 2016

Accepted Aug 22, 2016

Corresponding author Eun-Cho Park

E-mail encho76@naver.com only on the elderly but also on society as a whole. In order to alleviate this toll, solutions to function degradation in the elderly population must be found. ${ }^{3}$

Aging causes functional and structural changes in elderly people. These changes cause muscle weakness that results from a reduction of muscle area and size, which leads to a decline in physical function. ${ }^{4,5}$ Physical and functional changes in the elderly affect their ability to carry out daily activities. ${ }^{6}$ In particular, muscle weakness of the trunk and pelvic limbs and decreased proprioceptive sensibility might hamper mobility and balance. ${ }^{7}$ These changes increase the risk of injury from falls. ${ }^{8}$

Balance can be divided into static balance and dynamic balance. Static balance is the ability to maintain center of gravity and the same posture so that the body does not move. Dynamic balance is the ability to maintain a desired posture by maintaining center of

Copylight (C2016 The Korea Society of Physical Therapy

This is an Open Access article distribute under the terms of the Creative Commons Attribution Non-commercial License (Http:// creativecommons.org/license/by-nc/4.o.) which permits unrestricted non-commercial use, distribution, and reproduction in any medium, provided the original work is properly cited. 
gravity while the body is moving. ${ }^{9}$ Maintaining balance requires vestibular function, visual information, proprioceptive sense, musculoskeletal function, stability of the trunk, and cognitive ability. Aging deteriorates these functions and can lead to falls. ${ }^{10}$

Many forms of exercise, such as muscle strengthening exercise, flexibility exercise, and aerobic exercise, can enhance the balance of elderly people and help them maintain or build muscle mass. The usefulness of these exercises has been proven. ${ }^{11}$ The trunk stabilization exercise based on the strengthening of the core muscle for static body position. ${ }^{12}$ Hess and Woollacott ${ }^{13}$ said that high-intensity muscle strengthening exercise is effective for elderly people whose balance ability is impaired and that this exercise effectively reduces the risks of getting hurt from a fall. Horak et al. ${ }^{14}$ said that the elderly use a hip strategy to keep balance, and since this hip strategy uses proximal muscles like abdominal muscles, and Granacher et al. ${ }^{15}$ said that trunk muscle strength are associated with variables of static/dynamic balance, functional performance, and falls. Hodges and Richardson ${ }^{16}$ conducted a study using an electromyogram and found that trunk muscle activities are essential for maintaining the stability of the lumbar and that transverse abdominal muscle and the multifidus muscle each have shorter a muscle contraction on-set time than the muscles that move the limbs. They also found that the contraction of trunk muscles, such as the musculus transversus abdominis, and the stability of the trunk, which refers to a person's ability to control the movement of joints both consciously and unconsciously, are important for a person's ability to move in a certain way. ${ }^{17}$ In addition, they also found that the ability to control one's head is important for controlling posture and balance. ${ }^{18}$ Gorden and $\mathrm{Ghez}^{19}$ emphasized that while the neck muscles are shorter and thicker than other muscles, the neck muscles have the highest concentration of muscle spindle out of all human muscles and exchange information through nervous system networks, such as the vestibulospinal reflex, the vestibulocollic reflex, and the vestibuloocular reflex, which control the arrangement of the head and trunk. Neck muscles help to support the head in a stable way, to hold one's gaze, ${ }^{20}$ and to maintain whole-body activity. ${ }^{21}$ Based on the findings of these previous studies, the stability of the trunk and neck are clearly important for maintaining balance and posture, and both trunk stabilization and neck stabilization exercises are required to enhance the balance of the elderly.

A limitied number of studies emphasize the effect of neck, trunk stability on posture control. Research into the correlation of neck stability with trunk is currently insufficient especially for elderly people. Therefore, studies on the impact of trunk and neck stabilization exercises on the static and dynamic balance of the elderly need to be conducted in order to fill a gap in knowledge on the subject.

\section{METHODS}

\section{Subjects}

A total of 30 senior citizens who were over 65 years old from the $\mathrm{K}$ Senior Welfare Center in K City were selected for the study. Each participant was randomly allocated to either the neck and trunk stabilization exercise group (NTSG) $(\mathrm{n}=15)$ or the trunk stabilization exercise group (TSG) $(n=15)$. The selection standards for the participants were those who had not fallen over during the previous year, those who had not suffered serious pain and musculoskeletal or neurological damage, those who were not on medication that might impair balance, those who had not participated in a regular trunk or neck stabilization exercise, those who were capable of independent walking, those whose Korean Mini-Mental State Examination (K-MMSE) score was over 24, and those who fully understood the purpose of this study. The general characteristics of the participants are listed in the Table 1.

This study was performed in compliance with the ethical principles of the Declaration of Helsinki. The subjects agreed to participate in the study after receiving explanations of the purpose and procedures of the experiment, and they signed an informed consent statement before participation. The protocol for this study was approved by the ethics committee.

\section{Experimental methods}

\section{1) Interventions}

For the trunk stabilization exercise, three exercises were conducted.

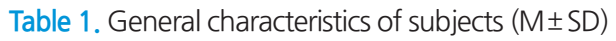

\begin{tabular}{lcccc}
\hline & NTSG $(n=15)$ & TSG $(n=15)$ & $t$ & $p$ \\
\hline Gender (male/female) & $6 / 9$ & $6 / 9$ & & \\
Age (year) & $73.4 \pm 5.8$ & $74.7 \pm 7.8$ & -0.531 & 0.60 \\
Height (cm) & $163.4 \pm 7.3$ & $166.8 \pm 5.3$ & -1.485 & 0.15 \\
Weight (kg) & $62.2 \pm 7.9$ & $63.4 \pm 6.0$ & -0.463 & 0.65 \\
MMSE (score) & $28.8 \pm 1.3$ & $28.5 \pm 1.2$ & 0.744 & 0.46 \\
\hline
\end{tabular}

$\mathrm{M} \pm$ SD: mean \pm standard deviation, NTSG: neck and trunk stabilization exercise group, TSG: trunk stabilization exercise group. 
First, a participant lay back with their knees bent and tightened their stomach by pressing the navel down. Second, they put their knees and feet apart at the shoulder width, put their feet on the floor, raised the pelvis off the floor to form a straight line from the knees to the chest, paused, and slowly pulled the pelvis down. Third, they lay back with their knees bent, lifted the feet about $10 \mathrm{~cm}$ off the floor, tried to stick their shoulder on the floor, kept the trunk, and slowly moved the pelvic limbs from side to side..$^{22,23}$

For the trunk and neck stabilization exercise, each participant was asked to conduct craniocervical flexion training while carrying out the trunk exercises in a supine position to contract their deep neck flexor muscles when their trunk muscles were contracted. For the craniocervical flexion training, a pressure biofeedback unit (PBU) (Chattanooga, USA) that was filled with air was placed on the back of the head and upper neck of each participant. The pressure gauge was set to $20 \mathrm{mmHg}$, and the participants were fully relaxed. The superficial muscle of the neck remained relaxed and their deep neck flexors were contracted to flex the craniocervical region so that the cervical lordosis would be reduced. The jaw was drawn and the head was pushed down to the floor so the gauge of the pressure biofeedback unit went up. The pressure gauge of the PBU was checked and the participant's neck was laid down flat. The pressure was increased by $2 \mathrm{mmHg}$ from $22 \mathrm{mmHg}$ to $30 \mathrm{mmHg}^{24,25}$

Both groups received training for 30 minutes per day three times per week for eight weeks. A preliminary evaluation of each patient was conducted before starting the program and reevaluation was conducted after eight weeks.

\section{2) Measures}

\section{(1) Balance analysis system}

To measure balance, a biofeedback analysis system (AP1153 Biorescue, France), which measures the static and dynamic balance of patients, healthy people, and athletes, was used. The participants were asked to stand and keep their eyes forward, were informed of the measuring method through a monitor, and were shown a demonstration before the measurement was taken. The participants were asked to stand for one minute as they maintained balance and kept their eyes forward to measure sway length based on pressure. They maintained balance by using an ankle strategy, following directions from the monitor. Then anterior limit of stability and posterior limit of stability were measured to compare balance.

\section{(2) The timed up and go test}

The timed up and go (TUG) test is a method used to measure a person's basic mobility and balance by measuring the amount of time it takes a person to go from a sitting to standing position, walk three meters, turn around, return to the chair, and sit back down. Studies say that this series of activities usually takes less than 10 seconds for healthy adults and 11-20 seconds for the feeble elderly or the elderly with disabilities. A person who takes more than 20 seconds to complete these activities is regarded as having a functional movement impairment. The interrater reliability of the study was $r=0.99$ and the intrarater reliability was $r=0.98 .{ }^{26}$ The measurements of the TUG test were made three times to calculate the average results.

\section{(3) Pressure biofeedback unit (PBU)}

A PBU (Chattanooga Group Inc., Hixson, USA) was used for the neck stabilization exercise. The PBU consisted of a measuring instrument, an air bag, and pneumatic pumps.

\section{Statistical analysis}

Statistical processing of the data was conducted using SPSS ver. 12.0, and descriptive statistics were used to analyze the subjects' general characteristics. An independent t-test was used to examine the homogeneity of the participants. Two-way repeated ANOVA was used to compare of balance ability according to training period and type on groups. Training group was set as the factor of between-subject, and Period was set as the factor of within-subject. And Variables of balance ability were set dependent variable. An independent t-test was conducted to examine the differences between the two groups. A statistical significance level was set at $\mathrm{p}<0.05$.

\section{RESULTS}

In results of two-way repeated ANOVA to compare of balance ability according to training period and type on groups, for sway length, anterior limit of stability, posterior limit of stability and TUG, there were no significant difference in between-subjects effect $(p>0.05)$. But, there were significant difference in within-subject effect according to period and in interaction of group and period (Table 2). For sway length, anterior limit of stability, posterior limit of stability and TUG, two-way repeated ANOVA did'nt show significant effects of group $(\mathrm{p}>0.05)$. But, two-way repeated ANOVA showed signifi- 
Table 2. Comparison of balance ability according to training period and type on groups (M $\pm S D)$

\begin{tabular}{|c|c|c|c|c|c|c|}
\hline & & Pre & Post & Difference Value & & $F(p)$ \\
\hline \multirow[t]{4}{*}{ Sway Length $(\mathrm{cm})$} & NTSG & $36.10 \pm 17.51$ & $28.39 \pm 9.89$ & $-7.71 \pm 10.45$ & $\mathrm{G}^{\mathrm{a}}$ & $0.04(0.843)$ \\
\hline & TSG & $33.90 \pm 10.66$ & $32.32 \pm 9.84$ & $-1.58 \pm 2.63$ & $\mathrm{~Pb}^{\mathrm{b}}$ & $11.143\left(0.002^{*}\right)$ \\
\hline & $\mathrm{t}$ & 0.416 & -1.090 & -0.202 & \multirow{2}{*}{$P x G^{c}$} & \multirow{2}{*}{$4.85\left(0.036^{*}\right)$} \\
\hline & $\mathrm{p}$ & 0.681 & 0.285 & $0.043^{*}$ & & \\
\hline \multirow[t]{4}{*}{ Anterior LOS (mm) } & NTSG & $2,590.27 \pm 2,592.42$ & $6,119.20 \pm 3,565.39$ & $3,528.93 \pm 2,510.22$ & G & $1(0.326)$ \\
\hline & TSG & $2,556.60 \pm 2,685.39$ & $4,022.47 \pm 3,438.39$ & $1,465.87 \pm 1,604.18$ & $P$ & $42.168\left(<0.000^{*}\right)$ \\
\hline & $\mathrm{t}$ & 0.035 & 1.639 & 2.682 & \multirow{2}{*}{$P \times G$} & \multirow{2}{*}{$7.194\left(0.012^{*}\right)$} \\
\hline & $\mathrm{p}$ & 0.972 & 0.112 & $0.012^{*}$ & & \\
\hline \multirow[t]{4}{*}{ Posterior LOS (mm) } & NTSG & $1,761.53 \pm 1,700.71$ & $2,607.60 \pm 2,023.58$ & $846.07 \pm 778.93$ & G & $1.201(0.283)$ \\
\hline & TSG & $1,376.73 \pm 1,093.44$ & $1,773.73 \pm 1,204.27$ & $397.00 \pm 327.06$ & $P$ & $32.476\left(<0.000^{*}\right)$ \\
\hline & $\mathrm{t}$ & 0.737 & 1.371 & 2.059 & \multirow{2}{*}{ PxG } & \multirow{2}{*}{$4.238\left(0.049^{*}\right)$} \\
\hline & $\mathrm{p}$ & 0.467 & 0.181 & $0.049^{*}$ & & \\
\hline \multirow[t]{4}{*}{ TUG (sec) } & NTSG & $17.37 \pm 6.61$ & $7.61 \pm 1.24$ & $-9.76 \pm 6.29$ & G & $0.697(0.411)$ \\
\hline & TSG & $14.02 \pm 3.09$ & $9.19 \pm 1.74$ & $-4.83 \pm 3.03$ & $P$ & $65.411\left(<0.000^{*}\right)$ \\
\hline & $\mathrm{t}$ & 1.776 & -2.872 & -2.736 & \multirow{2}{*}{ PxG } & \multirow{2}{*}{$7.485\left(0.011^{*}\right)$} \\
\hline & $\mathrm{p}$ & 0.087 & $0.008^{\star}$ & $0.011^{*}$ & & \\
\hline
\end{tabular}

M \pm SD: mean \pm standard deviation, NTSG: neck and trunk stabilization exercise group, TSG: trunk stabilization exercise group, LOS: limit of stability, TUG: timed up and go test, G: Group, P: period.

${ }^{a}$ Between-subjects effect; ' $W$ ithin-subjects effect; ' Interaction effect of group and period. ${ }^{*} \mathrm{p}<0.05$.

cant effects of period and significant interaction of group and period. In results of independent t-test, significant differences were found between the two groups $(\mathrm{p}<0.05)$ (Table 2$)$.

\section{DISCUSSION}

Aging is accompanied by degradation of the body, such as muscle atrophy, muscle weakness, and musculoskeletal system degradation, ${ }^{27}$ and this degradation causes reduced balance and difficulties carrying out daily activities. ${ }^{28}$ Many studies have been conducted on the musculoskeletal system to help the elderly maintain or enhance their balance. According to these studies, the reaction time of the ankle muscles, which maintain balance, of the elderly was longer than that of young people. A significant reduction in the muscle strength of the ankle has been observed among the elderly. In order to maintain balance, elderly people can carry out hip joint and trunk exercises. ${ }^{14}$ Hodges and Richardson, 16 and Choi et al. ${ }^{29}$ reported that trunk muscle strength is essential for the elderly in order to maintain body posture and plays a role in maintaining the stability of the spine, performing body functions, and moving the arms and legs.

The present study aimed to examine the impact of the trunk stabilization exercise and the combined neck and trunk stabilization exercise on the balance of the elderly. Park and Hwangbo ${ }^{30}$ reported that both trunk stabilization exercises using a sling and trunk stabilization exercises on mat significantly decreased the sway area and the sway length of the center of the pressure in a standing position of stroke patients. In a study of eldely women conducted by Choi et al., ${ }^{31}$ Functional Reach Test (FRT), One Leg Stand Test with Open Eye (OLSTOE), One Leg Stand Test with Closed Eye (OLSTCE), Timed Up and Go Test (TUG) and 6m Walking Test (6MWT) have significantly improved after a trunk stabilization exercise using swiss ball and core stabilization exercise were performed. In a study of stroke patients conducted by Yoon et al., ${ }^{32}$ more significant improvements were observed in an experiment group than in a control group in an modified Forward Reach Test after carrying out an abdominal draw-in maneuver. In the present study, significant improvements in sway length of center of pressure in the standing position, anterior limit of stability, and posterior limit of stability were found in participants who underwent the trunk stabilization exercise, and these findings were similar to those of previous studies. Since the TUG times of the participants were significantly reduced after the exercise was completed, the exercise likely enhanced the balance of the participants. Based on these findings, the trunk stabilization exercise can likely be used to enhance the static and dynamic balance of the elderly. 
External information from the surrounding environment and internal information from within the body are delivered to the trunk and neck, allowing the limbs, including the neck, to coordinate with each other. Among them, the head and neck set body standards for the surrounding environment and provide information that allows posture to be controlled. ${ }^{33}$ To enhance the mobility of the distal parts of the body, including the neck, the trunk stabilization exercise should be conducted. The significance of the trunk stabilization exercise has been confirmed by previous studies. ${ }^{34}$ Since the elderly experience degradation of general body structure and functions, the effects of which negatively affect their balance, recovering the stabilization and balance of the neck and trunk is important for the elderly. Since muscle strength and the stability of neck muscles are related to the stabilization of the trunk, it was believed that these two factors might influence the stabilization of the trunk and balance.

In this study, the NTSG showed more significant improvements in their static and dynamic balance than the TSG. In addition, the TUG time decreased significantly after the exercise. These findings, along with previous study results, support the idea that strengthening the neck muscles enhances the stability of the head, which has positive effects on people suffering imbalance due to a lack of body stability and mobility. ${ }^{35}$ In a previous study conducted by Falla, ${ }^{36}$ the neck stabilization exercise was carried out for those with neck pain and those who did not have neck pain in order to compare changes in neck pain and the cervical vertebral and thoracic vertebrae alignment. The results of this study showed that the neck stabilization exercise group reported enhanced neck muscle strength and sitting balance. Shim et al. ${ }^{37}$ said arranging body posture in the supine position, carrying out the neck stabilization exercise, flexing the front and side parts of the trunk, doing bridge posture, and carrying our exercises that include a pelvic inclination exercise in the sitting position activate various muscles in the trunk. Controlling posture requires support, stabilization, and balance. To maintain a proper body posture against gravity, the head, trunk, arms, and legs should cooperate with each other.

While many studies on the elderly reported that exercise improved their muscle strength, flexibility, balance, and mobility and therefore enhanced their ability to carry out daily activities, effective exercise programs have not been introduced to the elderly because of their tendency to perform only limited activities as they age. Since more than $50 \%$ of the elderly over 65 years old fear falling, they avoid active and functional activities. Considering these factors, it is believed that the trunk and neck stabilization exercises in the present study can be used to enhance the static and dynamic balance of the elderly. Many elderly people participated in the study because the exercises used in this study can easily be carried out in a lying position on a broad foundation, which prevents them from falling, at home by themselves. More studies need to be conducted not only on the balance of the elderly but also on their walking abilities and how to prevent their falls.

\section{REFERENCES}

1. Yang YS. A study on time use and roles of the older adults in aging society. Center for social welfare research Yonsei university. 2007;16:1-26.

2. Yoon JY. A study on the propose about a sporting equipment to improve muscle tone for The old-focusing on the muscle used for everyday living. Journal of the Korean Society of Design Culture. 2011;17(4):318-26.

3. Stel VS, Smit JH, Pluijim SMF et al. Balance and mobility performance as treatable risk factors for recurrent falling in older person. J Clin Epidemial. 2003;56(7):659-68.

4. Lee BO, Choi JH, Lee KM. Relationship between physical function and cognitive function in elderly women. The korean journal of physical education. 2007;45(2):485-95.

5. Lee NK, Lee MH. Effects of different chair heights on ground reaction force and trunk flexion during sit-to-stand in the elderly. J Kor Phys Ther. 2014;26(6):449-52.

6. Jang HJ, Kim SY, Kwon CS. Relationships between flexed posture, physical performance and psychosocial factors in elderly women. J Kor Phys Ther. 2014;26(5):358-64.

7. Hicks GE, Simonsick EM, Harris TB et al. Trunk muscle composition as a predictor of reduced functional capacity in the health, aging and body composition study the moderating role of back pain. J Gerontal. 2005; 60(11):1420-4.

8. Park SD, Kim JY, Yu SH et al. Comparison of balance and fall efficacy of virtual reality program in elderly women with fall experience. J Kor Phys Ther. 2014;26(6):430-5.

9. Ragnarsdottir M. The concept of balance. Physiotherapy. 1996;82(6): 368-75.

10. Shumway-Cook A, Baldwin M, Polissar NL et al. Predicting the probaility for falls in community-dwelling older adults. Phys Ther. 1997;77(8): 812-9.

11. Chandler JM, Hadley EC. Exercise to improve physiologic and functional performance in old age. Clin Geriatr Med. 1996;12(4):761-84.

12. Han KS, Nam HD, Kim KH. Core muscle strengthening effect during spine stabilization exercise. J Electr Eng Technol. 2015;10(6):2413-9.

13. Hess JA, Woollacott M. Effect of high-intensity strength-training on functional measures of balance ability in balance-impaired older adults. Journal of Manipulative and Physiological Therapeutics. 2005;28(8): $582-90$. 
14. Horak FB, Shupert CL, Mirka A. Component of postural dyscontrol in the elderly. Neurobiol aging. 1989;10(6):727-45.

15. Granacher U, Gollhofer A, Hortobagyi T et al. The importance of trunk muscle strength for balance, functional performance, and fall prevention in seniors: A systematic review. Sports Med. 2013;43(7):627-41.

16. Hodges PW, Richardson CA. Contraction of abdominal muscle associated with movement of the lower limb. Phys Ther. 1997;77(2):132-42.

17. Kim K, Kim EK, Lee DK. Effects of PNF patterns exercise on pain, functional disability and fear avoidance belief in chronic low back pain patients. J Kor Phys Ther. 2014;26(2):110-6.

18. Hong JS. Cerebral palsy treatment ideas. Gunja, Seoul. 2011.

19. Gordon J, Ghez C. Muscle receptor and spinal reflexes: The stretch reflex. In: Schwartz J, eds, Principles of neural science, 3rd ed, London, Prentice-Hall International, 1991:565-80.

20. Seo HJ, Lee MJ, Oh TY. The effects of neck strengthening exercise on postural control in sitting position during reaching in children with spastic diplegia. NEUROTHERAPY. 2012;16(1):17-24.

21. Freitas SMSF, Duarte M, Latash ML. Two kinematic synergies in voluntary whole-body movements during standing. Journal of neurophysiology. 2006;95(2):636-45.

22. Seo DK, Kwon OS, Kim JH et al. The effect of trunk stabilization exercise on the thickness of the deep abdominal muscles and balance in patients with chronic stroke. J Phys Ther Sci. 2012;24(2):181-5.

23. Chung EJ, Kim JH, Lee BH. The effects of core stabilization exercise on dynamic balance and gait function in stroke patients. J Phys Ther Sci. 2013;25(7):803-6.

24. Jull G, Falla D, Vicenzino B et al. The effect of therapeutic exercise on activation of the deep cervical flexor muscles in people with chronic neck pain. Man Ther. 2009;14(6):696-701.

25. Chang JS, Lee JH. Comparison of cervical flexor muscles thickness during cranial-cervical flexor exercise according to pressure levels and eye directions in healthy subjects. J Kor Phys Ther. 2015;27(1):50-4.

26. Podisiadlo D, Richardson S. The timed up \& go: A test basic functional mobility for frail elderly person. J Am Geriatr Soc. 1991;39(2):142-8.

27. Schlicht J, Camaione DN, Owen SV. Effect oc intense strength training on standing balance, walking speed and sit to stand balance, walking speed and sit to stand performance in older adults. J Gerontal A Biol Sci Med. 2001;56(5):281-6.

28. Hageman PA, Leibowitz JM, Blanke D. Age and gender effects on postural control measures. Arch Phys Med Rehabil. 1995;76(10):961-5.

29. Choi SJ, Bang DH, So HJ et al. Characteristics of the abdominal and neck flexor muscles of children with cerebral palsy. J Kor Phys Ther. 2014;26(6):453-8.

30. Han GS, So JM, Lee KJ et al. The effect of isotonic excercise on a change for extended strength ratio (ESR) according to a posture change for the aged a low back pain patients. Korean Journal of Sport Biomechenics. 2006;16(4):195-203.

31. Hong SL, Kang KH, Kim TK et al. Effects of lumbar stabilization exercise with rehabilitative ultrasound imaging on lumbar deep muscle activity and lumbar isometric strength in athletes with chronic low back pain. Journal of Sport and Leisure Studies. 2010;40(2):621-34.

32. Yoon MR, Choi HS, Shin WS. Effects of the abdominal drawing-in maneuver and the abdominal expansion maneuver on grip strength, balance and pulmonary function in stroke patients. J Kor Phys Ther. 2015; 27(3):147-53.

33. Keshner EA, Woollacott MH, Debu B. Neck, trunk and limb muscle responses during postural pertubations in human. Exp Brain Res. 1998; 71(3):455-66.

34. Oh JL. The effects of trunk muscle strength training on sitting balance of children with spastic cerebral palsy. J Kor Phys Ther. 2004;16(1):87-102.

35. Gudjonsdottir B, Mercer VS. Hip and spine in children with cerebral palsy: Musculoskeletal development and clinical implications. Pediatric Physical Therapy. 1997;9(4):179-85.

36. Falla DL, Jull GA, Hodges PW. Patients with neck pain demonstrate reduced electromyographic activity of the deep cervical flexor muscle during performance of the craniocervical flexion test. Spine (Phila Pa 1976). 2004;29(19):2018-14.

37. Shim HB, Cho HY, Choi WH. Effects of the trunk stabilization exercise on muscle activity in lumbar region and balance in the patients with hemiplegia. J Kor Phys Ther. 2014;26(1):33-40. 